\title{
The respiratory rehabilitation Maugeri network service reconfiguration after 1 year of COVID-19
}

\author{
Michele Vitacca, Piero Ceriana, Bruno Balbi, Claudio Bruschi, Maria Aliani, Mauro Maniscalco, \\ Francesco Fanfulla, Aldo Diasparra, Luigino Rizzello, Daniela Sereni, Antonio Spanevello
}

Department of Respiratory Rehabilitation, ICS Maugeri IRCCS, Pavia, Italy

\begin{abstract}
As part of the Italian Health Service the respiratory ICS Maugeri network were reconfigured and several in-hospital programs were suspended to be substituted by workforce and facilities reorganization for acute and post-acute COVID-19 care need. The present review shows the time course variation of respiratory ICS network in terms of admissions diagnosis and outcomes. A comparative review of the admissions and outcome measures data (anthropometric, admission diagnosis, provenience, comorbidities, disability, symptoms, effort tolerance, disease impact, length of stay and discharge destinations) over 1 year period (March 2020-March 2021) was undertaken and compared to retrospective
\end{abstract}

Correspondence: Michele Vitacca, Istituti Clinici Scientifici Maugeri IRCCS, Via S. Maugeri 4, 27100 Pavia, Italy.

E-mail: michele.vitacca@icsmaugeri.it

Contributions: All the authors contributed equally. All the authors have read and approved the final version of the manuscript and agreed to be accountable for all aspects of the work.

Ethical approval and consent to participate: The study was approved by the Istituti Clinici Scientifici (ICS) Maugeri Ethics Committee (CEC 2279; March 12 ${ }^{\text {th }}, 2020$ ). At admission to ICS institutions, patients give their informed consent for the scientific use of their data. As a retrospective analysis, the study was not registered.

Conflict of interest: The authors declare that they have no competing interests, and all authors confirm accuracy.

Acknowledgments: This work was supported by the "Ricerca Corrente" funding scheme from the Italian Ministry of Health.

Key words: COVID-19; complex rehabilitation; hospital service reconfiguration; patient discharge; acute rehabilitation.

Received for publication: 12 March 2021.

Accepted for publication: 28 April 2021.

${ }^{\circ}$ Copyright: the Author(s), 2021

Licensee PAGEPress, Italy

Monaldi Archives for Chest Disease 2021; 91:1843

doi: 10.4081/monaldi.2021.1843

This article is distributed under the terms of the Creative Commons Attribution Noncommercial License (by-nc 4.0) which permits any noncommercial use, distribution, and reproduction in any medium, provided the original author(s) and source are credited. data from a corresponding 1 year (March 2019-March 2020) period to determine the impact of the network relocation on the delivery of pulmonary specialist rehabilitation to patients with complex needs during the pandemic episode. One of the changes implemented at the respiratory Maugeri network was the relocation of the Pulmonary Rehabilitation units from its 351 beds base to a repurposed 247 beds and a reduction in total number of admitted patients ( $n=3912$ in pre-COVID time; $n=2089$ in post-COVID time). All respiratory diagnosis, except COVID sequelae, decreased (chronic respiratory failure-CRF, COPD, obstructive sleep apnoea syndrome-OSAS, interstitial lung disease-ILD, tracheostomized patients and other mixed diseases decreased of 734, $705,157,87,79$ and 326 units, respectively). During the pandemic time, 265 post COVID sequelae with CRF were admitted for rehabilitation $(12.62 \%)$, percentage of patients coming from acute hospital increased, LOS and NIV use remained stable while CPAP indication decreased. Disease impact, dyspnoea and effort tolerance as their improvements after rehabilitation, were similar in the two periods. Only baseline disability, expressed by Barthel index, seems higher in the $2^{\circ}$ observation time as its improvement. Hospital deaths and transfers to acute hospitals were higher during pandemic crisis while home destination decreased. This review demonstrated impact of coronavirus pandemic situation, specifically the relocation of the respiratory inpatient rehabilitation wards in a huge Italian network.

\section{Introduction}

The global outbreak of coronavirus disease (COVID-19) is having a widespread diffusion throughout the world $[1,2]$. Italy, and particularly the northern region of Lombardy $[3,4]$ have been affected by an exponential growth of cases, which made necessary, for national and regional authorities, to implement extraordinary measures to contain the spread of the virus very quickly. Facilities reconfiguration included closure and relocation of some ward services based on the emerging evidence that some conditions increase the vulnerability to contracting and developing severe form of the COVID-19 disease. Implementation of this guidance impacted on the respiratory specialist rehabilitation service that provides multidisciplinary rehabilitation to patients with complex clinical conditions and disabilities [5]. In order to lighten the burden on acute care hospitals, certain rehabilitation facilities have been used to host COVID-19 patients in the post-acute phase of the disease, in particular involving respiratory rehabilitation departments with expertise in the management of lung diseases [6]. This reorganization has showed up with the need to modify tasks, roles, and scheduling of the rehabilitation teams, albeit in 
the absence of indications and guidelines on the management of patients in the post-acute phase, and data on their characteristics and rehabilitation needs [5].

This descriptive review aims to explore and to measure the impact of relocation of the Specialist Pulmonary Rehabilitation service through a revision of routine outcome measures collected in an Integrated Health Care Record database. In addition, this review enabled a comparison of the length of stay and discharge destination for the periods covered.

\section{Materials and Methods}

\section{Patients}

This study was conducted on the Automated Integrated Health Care Record database of respiratory patients admitted for inpatient pulmonary rehabilitation over 1 year period (March 2020march 2021) and compared to retrospective data from a corresponding 1 year (March 2019-march 2020) to Respiratory ICS Maugeri network of 8 hospitals in Italy (Bari, Ginosa, Telese, Lumezzane, Tradate, Pavia, Veruno, Milano), referral institutions for pulmonary rehabilitation, diagnosis and care for post-acute and chronic subjects $[7,8]$. ICS Respiratory Maugeri network is composed by a huge Italian Rehabilitation Department oriented to intervention for diagnosis, therapy and rehabilitation with high specialization and multidisciplinary approach. Its activity is spread over 4 important Italian Regions as a clinical and scientific reference point. Particular propensity of the network is: i) care of patients with high disabilities and needs suffering from severe chronic and exacerbated respiratory failure; ii) care of all related sleep pathologies in terms of diagnostics and therapy. Patients were transferred to our Institutions within 30 days following the recovery from an acute relapse which had required either acute hospital care or home management by the general practitioner. All patients received their regular treatment according to current guidelines for their disease stage [9]. Admissions and outcomes data were collected in the first year of the ICS Maugeri network relocation, a period coinciding with the coronavirus pandemic waves in Italy. To contextualize this information, the data was compared with retrospective data from the corresponding period in 1 year before. Direct comparison of patient discharge data: allowed the team to examine length of stay and patient complexity in two equivalent periods. The ICS Maugeri network database was developed to collate inpatient case episodes for patients admitted to ICS Rehabilitation services. The rehabilitation network is served by 351 beds and with suitably equipped therapy gyms located adjacent to units.

\section{Measurements}

At admission the following data were collected: demographics, anthropometrics, number and diagnosis of comorbidities by the Cumulative Illness Rating Scale (CIRS) including the Comorbidity Index (CI) and the Severity Index (SI) [10], diagnosis and conditions (tracheostomized and under long MV weaning period), provenience (hospital or home), as well as length of stay (LOS) in referring hospitals, use of mechanical ventilation (CPAP or NIV), outcome destinations (home, nursing home, acute hospital or death). The following outcome measures were assessed:

i. Motor performance was assessed by the Barthel Index (BI) [11]. The total BI score ranges from 0 (maximum level of dependency) to 100 (complete autonomy). A score $\leq 70$ corresponds to severe dependency ii. Symptom impact by the COPD Assessment Test (CAT) [12]

iii. The lower extremity function was assessed by means of the Short Physical Performance Battery (SPPB) [13]. The total SPPB score ranges from 0 to 12 , being 0 maximum and 12 no disability

iv. Functional dyspnoea by Medical Research Council (MRC) scale [14]

v. Dyspnoea by the Italian version of Barthel index dyspnoea (Bid) [15]: the total Bid score ranges from 0 (no) to 100 (maximum level)

vi. Exercise tolerance was assessed by the Six Minute Walking Test (6MWT) [16] using the predicted values of Enright et al. [17]. The baseline value of patients unable to perform the test was considered as 0 for analysis.

\section{Pulmonary Rehabilitation}

A multidisciplinary program according to ERS/ATS for chronic respiratory diseases [9] and according to Italian Position Paper for COVID patients was applied in all centers involved [16]. Type, intensity, timing and modality of intervention were tailored to the individual patient according to age, clinical severity, length of immobilization, comorbidities, starting from a minimum of one, 20-min daily session up to two-three, 30-min daily sessions. Subjects were re-assessed on a daily base in order to adjust the type, intensity, timing and modality of the intervention. According to patient's individual conditions the program might include also nutritional and psychological assessment.

\section{Statistical analysis}

Data were expressed as mean and SD and binary outcomes were described as percentage (\%).

\section{Results}

Data was obtained from the records of a total of 6001 patients. ICS Maugeri network has undergone a profound organizational transformation imposed by the Italian health authorities: the consequence has been the conversion of rehabilitation beds into facilities dedicated to the acute and acute care management of COVID pneumonia as well as the use of rehabilitation personnel to manage these beds. For this reason, during the time course of COVID spread and according to local need both numbers of available beds $(-29.62 \%)$ and number of admitted patients for respiratory rehabilitation (-46.60\%) decreased dramatically (Table 1). Figure 1 shows geographical details of patient's admissions loss according to different network centers. The majority of patients were males, with age over 65 years with important comorbidities without differences between the two periods (Table 1). All diagnosis except COVID sequelae decreased as absolute numbers admitted while chronic respiratory failure $(\mathrm{CRF})$ and tracheostomized patients under mechanical ventilation (MV) increased as \% in the second observational time when compared to the first one (Table 1). In details CRF, chronic obstructive pulmonary disease (COPD), obstructive sleep apnoea syndrome (OSAS), interstitial lung disease (ILD), tracheostomized patients and other mixed diseases decreased of 734, 705, 157, 87, 79 and 326 units respectively. 265 post COVID sequelae with CRF were admitted for rehabilitation $(12.62 \%)$. During the pandemic time, percentage of patients coming from acute hospital increased, length LOS and NIV use remained stable while CPAP indication decreased. Disease impact, 
Table 1. Anthropometric and clinical characteristics of admitted patients.

Pre COVID-19 Post COVID-19

\begin{tabular}{lcc} 
Observation time, months & 12 & 12 \\
Patients, n & 3912 & 2089 \\
\hline Males, n (\%) & $2464(63.40 \%)$ & $1357(65.21 \%)$ \\
Age, y & $70.44 \pm 10.95$ & $69.82 \pm 11.74$ \\
\hline CIRS II & $4.27 \pm 2.12$ & $4.28 \pm 2.23$ \\
Hospital beds, n & 351 & 247 \\
\hline Diagnosis & & \\
CRF, n (\%) & $1714(43.81)$ & $980(46.91)$ \\
COPD n (\%) & $1009(25.79)$ & $304(14.55)$ \\
OSAS n (\%) & $202(5.16)$ & $45(2.15)$ \\
ILD n (\%) & $117(2.99)$ & $30(1.43)$ \\
Tracheostomized under MV, n (\%) & $224(5.72)$ & $145(6.94)$ \\
\hline COVID + CRF n (\%) & $0(0)$ & $265(12.68)$ \\
Others n (\%) & $646(16.51)$ & $320(15.31)$ \\
\hline Provenience & & \\
Acute hospital, n (\%) & $1220(31.21)$ & $894(42.81)$ \\
Home, n (\%) & $2691(68.79)$ & $1190(57.19)$ \\
LOS, days & $23.37 \pm 12.37$ & $23.84 \pm 16.14$ \\
Devices use & & \\
NIV n (\%) & $512(13.1)$ & $261(12.5)$ \\
CPAP n (\%) & $989(25.38)$ & $339(16.24)$ \\
\hline
\end{tabular}

Data shown as mean SD or \%. CIRS, cumulative illness rating scale; CRF, chronic respiratory failure; COPD, chronic obstructive pulmonary disease; OSAS, obstructive sleep apnoea syndrome; ILD, interstitial lung diseases; MV, mechanical ventilation; LOS, length of stay; NIV, non-invasive ventilation; CPAP, continuous positive airway pressure

Table 2. Outcomes measures.

\begin{tabular}{|c|c|c|}
\hline & Pre COVID-19 & Post COVID-19 \\
\hline Barthel index in & $68.5 \pm 29.1$ & $60.46 \pm 31.79$ \\
\hline Barthel index out & $79.8 \pm 25.9$ & $76.90 \pm 28.39$ \\
\hline Delta Barthel index & $9.69 \pm 16.56$ & $15.09 \pm 10.61$ \\
\hline CAT in & $21.5 \pm 6.61$ & $20.46 \pm 7.46$ \\
\hline CAT out & $15.03 \pm 6.31$ & $12.62 \pm 6.43$ \\
\hline Delta CAT & $-6.72 \pm 5.70$ & $-8.30 \pm 6.23$ \\
\hline SPPB in & $7.50 \pm 3.81$ & $6.98 \pm 4.25$ \\
\hline SPPB out & $8.99 \pm 3.32$ & $9.03 \pm 3.41$ \\
\hline Delta SPPB & $1.34 \pm 2.26$ & $1.26 \pm 1.80$ \\
\hline MRC in & $2.94 \pm 1.08$ & $2.89 \pm 1.21$ \\
\hline MRC out & $1.86 \pm 1.06$ & $1.70 \pm 0.97$ \\
\hline Delta MRC & $-1.10 \pm 0.94$ & $-1.26 \pm 0.80$ \\
\hline Barthel dyspnoea in & $30.72 \pm 18.96$ & $32.84 \pm 19.74$ \\
\hline Barthel dyspnoea out & $21.10 \pm 16.41$ & $21.24 \pm 15.93$ \\
\hline Delta Barthel dyspnoea & $-9.53 \pm 12.25$ & $-11.34 \pm 10.67$ \\
\hline $6 \mathrm{mWD}$, meters in & $250 \pm 136$ & $249 \pm 156$ \\
\hline $6 \mathrm{mWD}$, meters out & $327 \pm 123$ & $329 \pm 145$ \\
\hline Delta 6mWD & $76.92 \pm 74$ & $78.8 \pm 72$ \\
\hline Deaths, n (\%) & $63(1.6)$ & $46(2.2)$ \\
\hline Acute transfers, n (\%) & $130(3.31)$ & $167(7.99)$ \\
\hline Nursing home, n (\%) & $57(1.47)$ & $26(1.24 \%)$ \\
\hline Home, n (\%) & $3662(93.62)$ & $1850(88.50)$ \\
\hline
\end{tabular}

Data shown as mean SD or \%. CAT, COPD assessment test; SPPB, short physical performance battery; MRC, Medical Research Council; 6mWD, six minute walking distance. dyspnoea and effort tolerance as their improvements after rehabilitation were similar in the two periods (Table 2). Only baseline disability expressed by Barthel index seems higher in the $2^{\text {nd }}$ observation time as its improvement. As expected, hospital deaths and transfers to acute hospitals were higher during pandemic crisis while home destination decreased (Table 2).

\section{Discussion}

This review demonstrated impact of coronavirus pandemic situation, specifically the relocation of the respiratory inpatient rehabilitation units in a huge Italian network showing a dramatical and progressive decrease of rehabilitation accesses (-46\%) for classical respiratory diseases at the expense of the new post-COVID pathology. Severity, disability, and outcomes seem unchanged when compared to pre COVID time except for a mild increase in mortality and transfer need.

Also, before COVID pandemic time, there was a profound need for rehabilitation services due to the ageing population and growing prevalence of non-communicable diseases [18]. Globally, in $2019,2 \cdot 41$ billion individuals had conditions that would benefit from rehabilitation, contributing to 310 million YLDs. This number had increased by $63 \%$ from 1990 to 2019. Increasingly, the value of Rehabilitation is being recognized as an integral component of the patient journey in facilitating safe and effective discharge for the deconditioned and significantly deteriorated patient.

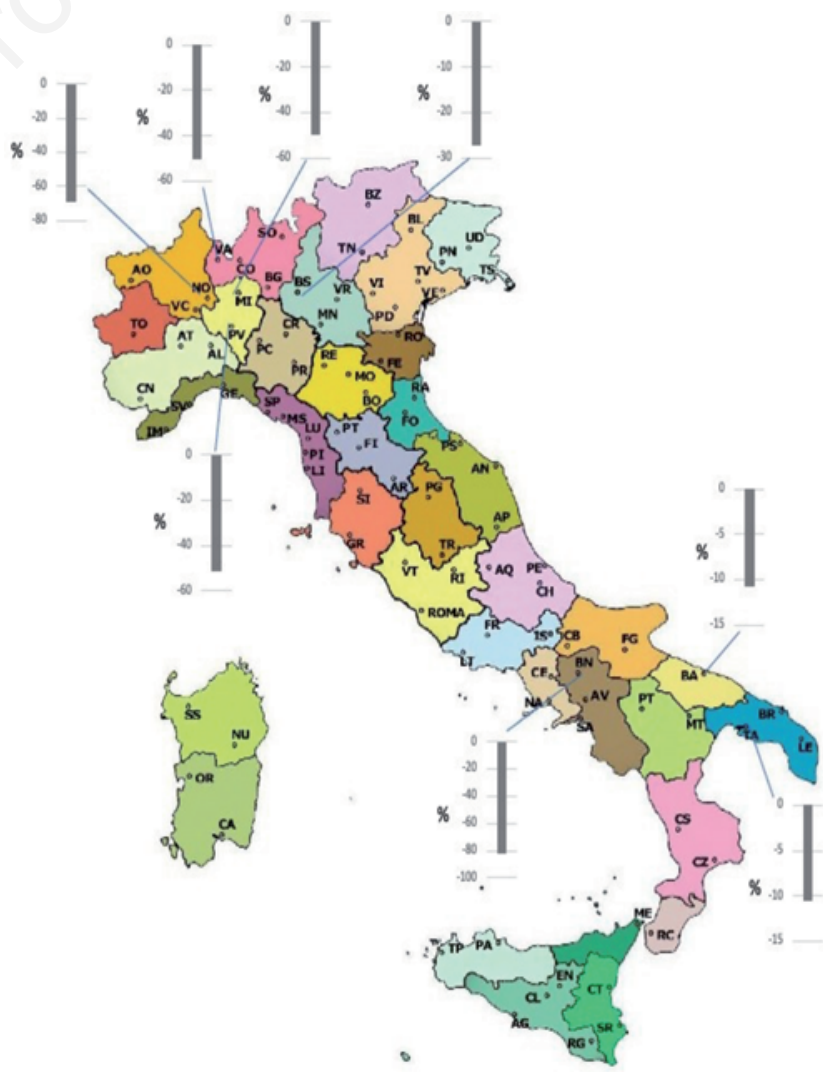

Figure 1. Geographical representation of patients' admissions loss (\%) among ICS Maugeri network [Lombardy Region: Veruno (NO), Tradate (VA), Milan, Pavia, Lumezzane (BS); and Apulia Region: Bari and Ginosa (TA)]. 
Global need for rehabilitation services was described as high, being at least one in every three people in the world needing rehabilitation in the course of their illness or injury [19].

The pandemic crisis has address long-standing structural disadvantages that expose chronic diseases to important restrictions as a huge impact both in terms of mortality and rehabilitation need. Short- term trends in excess deaths during the COVID-19 pandemic waves have not followed typical patterns for premature mortality in England and Wales particularly for deaths not directly attributable to the virus ranging from -1 per 100,000 to 26 per $100,000[19,20]$.

Stop of admissions to rehabilitation, early discharge and reduction of activities involved 194,800 inpatients in 10 countries. Outpatient activities stopped for $87 \%$, involving 318,000 patients per day in Italy, Belgium and the UK, leading to an estimate range of 1.3-2.2 million in Europe [21]. This fact may lead to future cumulative effects due to reduced functional outcome and consequent increased burden of care.

Within 2 months of COVID-19 being declared a pandemic, $41.2 \%$ of doctors reported cardiac rehabilitation services closure due to staff redeployment and facility closure [22]. Services who accepted referrals for medically high-risk patients pre-COVID-19 fell to only $43.5 \%$ during the COVID 19 pandemic while new reorganisation increased technology use and tele-rehabilitation.

Profound reorganizations for patients participating in cardiac, pulmonary, or vascular rehabilitation were to participate in homebased rehabilitation services in the setting of a temporary closure [23]. Approximately $70 \%$ of the patients were responsive to follow-up phone calls and were willing to track their individual progress in one or more areas. As a result, upon the re-opening of rehabilitation services, more than half of the patients returned to the center-based program [23].

Yet before COVID time, rehabilitation as a service was offered to only few people and specialist Rehabilitation beds were a finite resource under increasing demand in Italian hospitals [24,25].

In order to lighten the burden on acute care hospitals, our rehabilitation facilities have been used to host COVID-19 patients in the post-acute phase of the disease $[4,5,26]$.

The present data on a huge number of patients have confirmed that relocation of ICS Maugeri network rehabilitative service to care epidemic COVID waves unfortunately impacted the delivery of rehabilitation paths notably through the loss of the dedicated beds and patient's admission $[5,6,26]$.

It is interesting to note that the loss of admissions ranged from 10 to $82 \%$ according to local situation, restrictions, epidemiological course and health payers' decisions (Figure 1). The patients able to be admitted to rehabilitation paths in the COVID era were substantially similar for admission severity and disability to patients admitted in the pre-COVID time. The important novelty of this study is the information that COPD, OSAS and ILD were the diseases more penalized while patients with CRF with and without MV increased rehabilitation admission at least in term of percentage. The $\%$ of patients with post COVID sequalae represented about $12 \%$ of the total: this number seems not extremely high but clearly influenced by local differences and by more recent rehabilitation requests concentrated in the last months of 2020 and the beginning of 2021 .

It is noteworthy that rehabilitative outcomes (disability, effort tolerance, symptoms, disease impact) seem unchanged when compared to pre COVID time except for a mild increase for final deaths and transfer needs to acute hospitals probably related to a mild worsening in baseline disability and an increase in more complicated diseases as demonstrated by the higher number of CRF. Another important information from our study is that patients hos- pitalized in the second period improved their disability more than in the first period probably for the worse baseline level of disability at admission.

\section{Practical implications}

This study has discussed that in similar dramatic event in future, the needs of patients undergoing Rehabilitation should be a key consideration in the plans to relocate such services deliberating on service reconfiguration and relocation decisions for this essential service. Appropriate staff, tele rehabilitation programs, patient technology training, alternative methods of delivery for patients that do not have access to technology, as well as adequate reimbursements may be important recommendations. Developing policy that provides strategies to open closed rehabilitation network admitting high-risk/vulnerable populations, should be a national priority.

\section{Conclusions}

This service review has highlighted some of the impact on specialist inpatient rehabilitation facility at a large respiratory rehabilitative network in Italy showing a huge impact on people experiencing disability due to a reduction of all rehabilitation activities. It highlights the need for rehabilitation to be considered as a key component in the planning of the health care response to the pandemic similar future episodes.

\section{References}

1. Worldometer [Internet]. COVID-19 coronavirus pandemic. Accessed on: 10 April 2020. Available from: https://www.worldometers.info/coronavirus/

2. Global surveillance for human infection with coronavirus disease (COVID-2019). Accessed on: 10 April 2020. Available from: https://www.who.int/publicationsdetail/global-surveillance-for- human-infection-with-novelcoronavirus-(2019-ncov)

3. Istituto Superiore di Sanità. Accessed on: 10 April 2020. Available from: https://www.epicentro.iss.it/coronavirus5

4. Vitacca M, Migliori GB, Spanevello A, et al. Management and outcomes of post-acute COVID-19 patients in Northern Italy. Eur J Intern Med. 2020;78:159-160.

5. Simonelli C, Paneroni M, Fokom AG, et al. How the Covid-19 infection tsunami revolutionized the work of respiratory physiotherapists: An experience from northern Italy. Monaldi Arch Chest Dis 2020;90:1085.

6. Vitacca M, Migliori GB, Spanevello A, et al. Management and outcomes of post-acute COVID-19 patients in Northern Italy. Eur J Intern Med 2020;78:159-60.

7. Ceriana $P$, Nava S, Vitacca $M$, et al. Noninvasive ventilation during weaning from prolonged mechanical ventilation. Pulmonology 2019;25:328-33.

8. Maestri R, Bruschi C, Fracchia C, et al. Physiological and clinical characteristics of patients with COPD admitted to an inpatient pulmonary rehabilitation program: A real-life study. Pulmonology 2019;25:71-8.

9. Holland AE, Spruit MA, Troosters T, et al. An official 
European Respiratory Society/American Thoracic Society technical standard: field walking tests in chronic respiratory disease. Eur Respir J 2014;44:1428-46.

10. Linn BS, Linn MW, Gurel L. Cumulative illness rating scale. J Am Geriatr Soc 1968;16:622-6.

11. Mahoney FI, Barthel D. Functional evaluation: The Barthel Index. Md State Med J 1965;14:56-61.

12. Jones PW, Harding G, Berry P, et al. Development and first validation of the COPD Assessment Test. Eur Respir J 2009;34:648-54.

13. Bernabeu-Mora R, Medina-Mirapeix F, Llamazares-Herrán E, et al. The Short Physical Performance Battery is a discriminative tool for identifying patients with COPD at risk of disability. Int J Chron Obstruct Pulmon Dis 2015; 0:2619-26.

14. Fletcher CM. Standardised questionnaire on respiratory symptoms: a statement prepared and approved by the MRC Committee on the Aetiology of Chronic Bronchitis (MRC breath-lessness score). Br Med J 1960;2:1665.

15. Vitacca M, Paneroni M, Baiardi P, et al. Development of a Barthel Index based on dyspnoea for patients with respiratory diseases. Int J Chron Obstruct Pulmon Dis 2016;11:1199-206.

16. Vitacca M, Carone M, Clini EM, et al. Joint statement on the role of respiratory rehabilitation in the COVID-19 crisis: The Italian position paper. Respiration 2020;99:493-9.

17. Enright PL, Sherrill DL. Reference equations for the sixminute walk in healthy adults. Am J Respir Crit Care Med 1998;158:1384-7.

18. Kamenov K, Mills JA, Chatterji S, Cieza A. Needs and unmet needs for rehabilitation services: a scoping review. Disabil Rehabil 2019;41:1227-37.
19. Cieza A, Causey K, Kamenov K, et al. Global estimates of the need for rehabilitation based on the Global Burden of Disease study 2019: a systematic analysis for the Global Burden of Disease Study 2019. Lancet 2021;396:2006-17.

20. Kontopantelis E, Mamas MA, Deanfield J, et al. Excess mortality in England and Wales during the first wave of the COVID-19 pandemic. J Epidemiol Community Health 2021;75:213-23.

21. Negrini S, Grabljevec K, Boldrini P, et al. Up to 2.2 million people experiencing disability suffer collateral damage each day of COVID-19 lockdown in Europe. Eur J Phys Rehabil Med 2020;56:361-5.

22. Marzolini S, Kin R, Lima de Melo Ghisi G, et al. Cardiac rehabilitation in Canada during COVID- 19. CJC Open 2021;3:152-58.

23. Riley H, Stabile L, Wu WC. Transition to home-based treatment plans for center-based cardiac, pulmonary, and vascular rehabilitation during COVID-19. R I Med J (2013) 2020;103:30-3.

24. Vitacca M. [Lo stato della Riabilitazione Respiratoria in Italia].[Article in Italian]. Rassegna Patologia Apparato Respiratorio 2016;31:175-7.

25. Vitacca M, Scartabellati A, Banfi P, et al. [Malattie respiratorie in Lombardia: carotaggio AIPO su due settimane di attività].[Article in Italian]. Rassegna Patologia Apparato Respiratorio 2019;34:191-200.

26. Vitacca M, Crisafulli E, Balbi B, Carone M. [Ruolo della riabilitazione respiratoria nell'emergenza coronavirus pneumologia riabilitativa e assistenza domiciliare].[Article in Italian]. Rassegna Patologia Apparato Respiratorio 2020;3581-3. 